\title{
Fabrication of nitric oxide-releasing polyurethane glucose sensor membranes
}

\author{
Ahyeon Koh, Daniel A. Riccio, Bin Sun, Alexis W. Carpenter, Scott P. Nichols, and Mark H. \\ Schoenfisch $^{\star}$ \\ Department of Chemistry, University of North Carolina at Chapel Hill, Chapel Hill, NC, 27599, \\ USA
}

\begin{abstract}
Despite clear evidence that polymeric nitric oxide (NO) release coatings reduce the foreign body response (FBR) and may thus improve the analytical performance of in vivo continuous glucose monitoring devices when used as sensor membranes, the compatibility of the NO release chemistry with that required for enzymatic glucose sensing remains unclear. Herein, we describe the fabrication and characterization of NO-releasing polyurethane sensor membranes using NO donor-modified silica vehicles embedded within the polymer. In addition to demonstrating tunable $\mathrm{NO}$ release as a function of the NO donor silica scaffold and polymer compositions and concentrations, we describe the impact of the NO release vehicle and its release kinetics on glucose sensor performance.
\end{abstract}

\section{Keywords}

Nitric oxide release; Glucose biosensor; Polyurethane membrane

\section{Introduction}

\begin{abstract}
Strict monitoring and control of blood glucose levels are essential for effective diabetes management (Wang, 2008). Since conventional glucose monitoring (e.g., the finger prick method) provides only discrete snapshots of blood glucose levels, analytical methods that enable continuous monitoring of glucose concentration fluctuations via implantable enzymebased electrochemical sensors have been sought to improve diabetes management (Heller and Feldman, 2008; Klonoff, 2005b; Ward et al., 2002). Despite US Food and Drug Administration approval for certain devices, most sensors have serious shortcomings including poor accuracy, unpredictable signal stability, lag time in sensor response, frequent calibration requirement, and short lifetimes that limit their clinical utility (Klonoff, 2005a,b) Undesirable sensor performance has been attributed to the foreign body response (FBR) and biofouling (Wisniewski et al., 2000; Wisniewski and Reichert, 2000). As the outer surface of the sensor influences the FBR directly, recent work has focused on the development of more biocompatible sensor membranes to mitigate the FBR (Wilson and Gifford, 2005; Wilson and $\mathrm{Hu}, 2000)$. The general consensus is that new strategies to improve long-term in vivo sensor performance should: (1) reduce the initial inflammatory response; (2) enhance wound healing; and, (3) avoid biosensor degradation (Gifford et al., 2005).
\end{abstract}

\footnotetext{
(C) 2011 Elsevier B.V. All rights reserved.

* Corresponding author. Tel.: +1 919843 8714; fax: +1 919962 2388. schoenfisch@ unc.edu (M.H. Schoenfisch).
}

Appendix A. Supplementary data: Supplementary data associated with this article can be found, in the online version, at doi:10.1016/ j.bios.2011.06.005. Figures and tables of supporting data are indicated with the notation ' $\mathrm{S}$ ' in the results and discussion. 
Due to nitric oxide's (NO) role as an endogenous antibacterial agent (Williams, 2003), we reported on the synthesis of sol-gel-derived coatings (e.g., xerogels) capable of storing and spontaneously releasing NO (Hetrick and Schoenfisch, 2006; Nablo et al., 2005b; Riccio et al., 2009). These materials were shown to reduce bacterial adhesion (Nablo et al., 2001; Nablo and Schoenfisch, 2003), kill bacteria that did manage to adhere (Hetrick and Schoenfisch, 2007; Hetrick et al., 2008; Privett et al., 2010), and reduce in vivo infection rates (Nablo et al., 2005a). Furthermore, the NO release was shown to reduce collagen capsule thickness around a subcutaneous implant by $>50 \%$ relative to controls in a rat model (Hetrick et al., 2007). Concomitantly, the NO-releasing implant lessened the chronic inflammatory response at the tissue/implant interface by $>30 \%$ and enhanced blood vessel formation by $>77 \%$ versus controls (Hetrick et al., 2007). Nitric oxide-releasing sensors have exhibited improved biocompatibility and sensor performance (Gifford et al., 2005; Yan et al., 2011). For example, Gifford et al. reported reduced inflammatory response for subcutaneously implanted NO-releasing glucose sensors over 3 d (Gifford et al., 2005). In blood, Yan et al. described improved hemocompatibilty and in vivo sensor performance for an intravenous NO-releasing glucose/lactate sensor (Yan et al., 2011). We recently reported enhanced in vivo glucose recovery from NO-releasing microdialysis probes during longterm (i.e., 2 weeks) implantation (Nichols et al., 2011). Collectively, these studies indicate that NO release is a highly promising strategy that may solve the lingering biocompatibility problems encountered when glucose biosensors are implanted in vivo.

Based on the reduced FBR as a function of NO release, we sought to combine the chemistries of NO release with enzymatic glucose sensing to fabricate continuous glucose monitoring devices with improved biocompatibility and extended analytical performance. Although promising with respect to reduced capsule formation and implant-associated infection, the xerogel films resulted in unusually low glucose response when used to fabricate glucose biosensors (Shin et al., 2004). The glucose impermeability of the xerogel film was attributed to enhanced hydrolysis and condensation rates catalyzed by the aminosilanes and exposure to high pressures of $\mathrm{NO}$ (necessary to form the diazeniumdiolate-based NO donors). To address the inadequate glucose permeability, we fabricated functional NO-releasing glucose biosensors by patterning xerogel microarrays on top of (Oh et al., 2005) or physically entrapping ground xerogel particles within (Shin et al., 2004) standard polyurethane sensor membranes. These polymer configurations allowed for enhanced glucose sensitivity relative to sensors completely coated with xerogel films because significant potions of the underlying electrode surface remained unmodified by the xerogel.

To date, a key challenge for the development of NO-releasing glucose biosensors has been limited NO release. While our previously published hybrid NO-releasing xerogel particle/ polyurethane biosensor exhibited excellent analytical response characteristics (e.g., sensitivity, linear response, and lifetime), the NO flux and release duration were limited to a maximum of $\sim 90 \mathrm{pmol} \mathrm{cm}^{-2} \mathrm{~s}^{-1}$ for $2 \mathrm{~d}$ (Shin et al., 2004). The sensors described more recently by Gifford et al. and Yan et al. released $\mathrm{NO}$ with average maximum fluxes of 7.52 pmol cm$~^{-2} \mathrm{~s}^{-1}$ and $40 \mathrm{pmol} \mathrm{cm}^{-2} \mathrm{~s}^{-1}$ for $18 \mathrm{~h}$ and $7 \mathrm{~d}$, respectively (Gifford et al., 2005; Yan et al., 2011). As such, a void exists regarding the optimal level and duration of NO release required to mitigate the FBR and influence sensor performance. Herein, we report the synthesis of NO-releasing glucose sensor membranes with a wide range of NO fluxes (5 pmol cm $\mathrm{cm}^{-2} \mathrm{~s}^{-1}$ to $2.5 \mathrm{nmol} \mathrm{cm}-2 \mathrm{~s}^{-1}$ ) and release durations ( $16 \mathrm{~h}$ to $14 \mathrm{~d}$ ) using NO donormodified silica nanoparticles. The NO-releasing polymers are then used to fabricate functional glucose biosensors to further evaluate sensor response and stability as a function of NO release. 


\section{Materials and methods}

\subsection{Reagents and materials}

Glucose oxidase (type VII from Aspergillus niger), hydrogen peroxide (30\% v/v), L-proline, and $\beta$-D-glucose anhydrous were purchased from Sigma (St. Louis, MO). Ethanol (EtOH), tetrahydrofuran (THF), and aqueous ammonium hydroxide solution $(14.8 \mathrm{M})$ were purchased from Fisher Scientific (Pittsburgh, PA). Methyltrimethoxysilane (MTMOS) was purchased from Fluka (Buchs, Switzerland). $N$-(6-aminohexyl)aminopropyltrimethoxysilane (AHAP3), $N$-(2-aminoethyl)-3-aminopropyltrimethoxysilane (AEAP3), 3methylaminopropyltrimethoxysilane (MAP3), 3-mercaptopropyltrimethoxysilane (MPTMS), tetramethoxysilane (TMOS), and tetraethoxysilane (TEOS) were purchased from Gelest (Tullytown, PA). Tecoplast TP-470-000, Tecophilic HP-93A-100, and Tecoflex SG-80A (TPU) were gifts from Thermedics (Woburn, MA). Hydrothane AL 25-80A (HPU) was a gift from AdvanSource Biomaterials Corporation (Wilmington, MA). A Griess reagent kit was purchased from Promega (Madison, WI). Water was purified $(18.2 \mathrm{M} \Omega / \mathrm{cm}$; total organic contents $<6 \mathrm{ppb}$ ) with a Milli-Q UV Gradient A10 system (Millipore Corp., Bedford, MA). Nitric oxide, nitric oxide calibration gas (26.4 ppm; balance N2), nitrogen and argon (Ar) gases were purchased from National Welders Supply (Durham, NC). All other reagents and solvents were analytical-reagent grade and used as received.

\subsection{Preparation of NO-releasing dopant}

1-[2-(carboxylato)pyrrolidin-1-yl]diazen-1-ium-1,2-diolate (PROLI/NO) was prepared by converting L-proline to NO donor form following a procedure reported by Saavedra et al. (1996). Nitric oxide-releasing silica particles were synthesized based on the sol-gel process via the co-condensation of an aminosilane (i.e., MAP3, AEAP3, or AHAP3) or mercaptosilane (i.e., MPTMS) in a range of concentrations (65-75 mol\%) with tetraethyoxysilane (TEOS) or tetramethoxysilane (TMOS) in similarly to those reported in previous studies (Riccio et al., 2011; Shin et al., 2007; Shin and Schoenfisch, 2008). Subsequent diazeniumdiolation of the amine-containing particles was performed under high pressure of $\mathrm{NO}$ for $3 \mathrm{~d}$ in the presence of sodium methoxide at room temperature.

Nitrosation of the thiol-containing nanoparticles was carried out by reaction with acidified nitrite in the dark at $0{ }^{\circ} \mathrm{C}$. The details of the NO-releasing characteristics and particle sizes for each particle system are provided in supporting information (Table S1).

\subsection{Hybrid sol-gel/polyurethane glucose sensor fabrication}

A platinum macroelectrode $\left(0.3 \mathrm{~cm}\right.$ radius and $0.031 \mathrm{~cm}^{2}$ platinum surface area) was used to fabricate hybrid sol-gel/polyurethane glucose biosensors that consisted of two distinct layers: glucose oxidase and NO-releasing polyurethane. Fabrication of the glucose sensor was adapted from a procedure described by Shin et al. (Shin et al., 2004). Briefly, glucose oxidase (GOx) was immobilized within a sol-gel matrix on a polished platinum electrode by casting $3 \mu \mathrm{L}$ of a GOx sol. The enzyme-containing sol was prepared by dissolving $9 \mathrm{mg}$ of GOx into $75 \mu \mathrm{L}$ of water and adding $50 \mu \mathrm{L}$ of this solution into $25 \mu \mathrm{L}$ MTMOS and $100 \mu \mathrm{L}$ EtOH followed by mixing for $10 \mathrm{~min}$. The NO-releasing layer was prepared by dispersing silica particles in a polyurethane (PU) polymer solution consisting of $20 \mathrm{mg} \mathrm{mL}^{-1} 50 \% \mathrm{w} / \mathrm{w}$ TPU and HPU dissolved in 50\% v/v THF/EtOH solution. The NO-releasing layer was formed by casting $5 \mu \mathrm{L}$ of the resulting $9-144 \mathrm{mg} \mathrm{mL}^{-1} \mathrm{NO}$-releasing particles in a PU polymer solution $\left(0.2-2.4 \mathrm{mg} \mathrm{cm}^{-2}\right)$. Particle concentration rather than casting volume was increased when increasing particle concentration per surface area. For instances where an additional polyurethane layer was cast on top of the NO-releasing layer, $20 \mu \mathrm{L}$ of $20-60 \mathrm{mg}$ $\mathrm{mL}^{-1}$ polyurethane polymer solution dissolved in THF was used and each layer was dried under ambient conditions for $30 \mathrm{~min}$ before casting the subsequent layer. 


\subsection{Film fabrication and methods of film study}

Glass substrates were prepared via sonication in $\mathrm{EtOH}$ and subsequent UV-ozone cleaning for $20 \mathrm{~min}$ in a BioForce Tip Cleaner (Ames, IA). The NO-releasing particle-dispersed polyurethane solution described previously was spread-cast $(36.2 \mu \mathrm{L})$ onto a glass substrate $\left(2.08 \mathrm{~cm}^{2}\right)$. For films with an additional PU layer on the NO-releasing layer, PU polymer solution was cast $(141 \mu \mathrm{L})$ in the same manner as the electrode casting procedure. Each layer was dried under ambient conditions for $30 \mathrm{~min}$ and stored in the dark at $-20{ }^{\circ} \mathrm{C}$ under vacuum until used. This storage method was adopted to assure NO donor stability. Although the $N$-diazeniumdiolated particle-doped films could be stored at ambient condition (humidity $40 \%$ at room temperature) without loss of NO for $2 \mathrm{~d}$, NO storage was sacrificed $(\sim 10 \%)$ under more humid conditions (i.e., $90 \%$ humidity, $23.7-25.0{ }^{\circ} \mathrm{C}$ ). Nitric oxide release from NO-releasing silica particle-doped polyurethane was characterized using a Sievers model 280i chemiluminesence NO analyzer (Boulder, CO). Films were immersed in $0.01 \mathrm{M}$ PBS (pH 7.4) buffer solution at $37^{\circ} \mathrm{C}$ sparged with $80 \mathrm{~mL} \mathrm{~min}{ }^{-1}$ nitrogen with additional nitrogen supported to the flask to match the instrument collection rate of $200 \mathrm{~mL}$ $\min ^{-1}$. The instrument was calibrated using $26.4 \mathrm{ppm} \mathrm{NO}$ gas (balance $\mathrm{N}_{2}$ ) and air passed through a Sievers NO zero filter. When measuring NO from nitrosothiol-based materials, the sample flask was shielded from light to prevent light-initiated NO release. Total NO release was determined spectrophotometrically by measuring the conversion of NO to nitrite $\left(\mathrm{NO}_{2}{ }^{-}\right)$using the Griess assay (Chae et al., 2004). After soaking NO-releasing films in PBS buffer solution at $37^{\circ} \mathrm{C}$ for a period exceeding their NO release, $50 \mu \mathrm{L}$ of the solution was mixed with $100 \mu \mathrm{L}$ of Griess reagent (1\% w/v sulfanilamide in $5 \% \mathrm{v} / \mathrm{v}$ phosphoric acid and $0.1 \% \mathrm{w} / \mathrm{v} N$-(1-naphthyl)ethylenediamine dihydrochloride) and incubated at room temperature for $10 \mathrm{~min}$. Absorbance of these solutions was measured at $540 \mathrm{~nm}$ using a Labsystem MultiskanRC microplate spectrophotometer (Helsinki, Finland). Total nitrite concentration was determined using a calibration curve constructed with a standard nitrite solution. The water uptake of polyurethane films was determined by measuring the mass of glass substrates cast with $141 \mu \mathrm{L}$ of $40 \mathrm{mg} \mathrm{mL}^{-1}$ polyurethane before/after soaking in PBS for $3 \mathrm{~h}$. The degree of silica particle leaching from the sensor membranes was assessed via dynamic light scattering (DLS). The NO-releasing polyurethane-coated glass substrates were immersed in $2 \mathrm{~mL}$ PBS and incubated at $37^{\circ} \mathrm{C}$ for $7 \mathrm{~d}$. The concentration of silica particles that leached into the soak solution was determined by measuring the derived count rate that varied linearly with particle concentration (Amstad et al., 2009; Lahtinen et al., 2010). The derived count rate was then fit to a calibration curve of known particle concentrations. Film thickness was measured with a Tencor Alpha-Step 200 Profilometer (San Jose, CA). Half of the NO-releasing silica particle-doped polyurethane coating was removed from the glass substrate and the resulting interface was probed to determine film thickness.

\subsection{Sensor performance of hybrid sol-gel/polyurethane glucose sensor}

The analytical performance (i.e., sensitivity, dynamic range, and response time $\left(t_{95 \%}\right)$ ) of the biosensors was evaluated via chronoamperometry using a CH Instruments 1030A potentiostat (Austin, TX). All electrochemical measurements were performed in $0.01 \mathrm{M}$ PBS ( $\mathrm{pH} 7.4)$ at room temperature using a three-electrode configuration with a $\mathrm{Ag} / \mathrm{AgCl}$ $(3.0 \mathrm{M} \mathrm{KCl})$ reference electrode, a platinum wire counter electrode, and a glucose biosensor as the working electrode. Glucose sensors were first hydrated in PBS for $1 \mathrm{~h}$ and polarized for $20 \mathrm{~min}$ to stabilize glucose response. Indeed, sensor response effects due to NO release and changes in membrane permeability (water uptake) were shown to significantly decrease during this preconditioning period (data not shown). Response and calibration curves were obtained by injecting $1 \mathrm{M}$ glucose aliquots into $30 \mathrm{~mL}$ PBS at room temperature under constant stirring and an applied potential of $+0.6 \mathrm{~V}$ vs. $\mathrm{Ag} / \mathrm{AgCl}$ (Schoenfisch et al., 2006; Shin et al., 2004). Permeability $\left(P_{i}^{e}\right)$ was defined electrochemically as the ratio of peak 
current at the hybrid sol-gel/polyurethane glucose sensor $\left(\Delta I_{\mathrm{x}}\right)$ and bare platinum electrode $\left(\Delta \mathrm{I}_{\mathrm{b}}\right)$ response at either $0.79 \mathrm{mM} \mathrm{H}_{2} \mathrm{O}_{2}\left(P_{\mathrm{H}_{2} \mathrm{O}_{2}}^{e}\right.$ ) or air saturated solution $\left(P_{\mathrm{O}_{2}}^{e}\right)$ (Schoenfisch et al., 2006; Shin et al., 2008). Amperometric selectivity coefficients were calculated using Eq. (1) (Shin et al., 2008) where $\Delta I_{\mathrm{Glu}}$ and $\Delta I_{\mathrm{j}}$ are the measured current values for glucose (Glu) and interfering species ( $\mathrm{j}=$ ascorbic acid, uric acid, and nitrite), respectively.

$$
\log K_{\mathrm{Glu}, \mathrm{j}}^{\mathrm{amp}}=\log \left(\frac{\Delta I_{\mathrm{j}} / c_{\mathrm{j}}}{\Delta I_{\mathrm{Glu}} / c_{\mathrm{Glu}}}\right)
$$

The concentration of each interfering substance $\left(c_{\mathrm{j}}\right)$ was $100 \mu \mathrm{M}$. The glucose concentration $\left(c_{\mathrm{Glu}}\right)$ employed for selectivity coefficient determination was $5.6 \mathrm{mM}$.

\section{Results and discussion}

\subsection{NO-releasing glucose sensor}

Nitric oxide-releasing glucose sensor membranes were prepared using polyurethanes doped with NO-releasing silica particles. These membranes were then used as the outermost coating for enzyme-based glucose sensors. Since NO release has previously been reported to increase the background current of amperometric glucose sensors because of the overlapping oxidation potentials of $\mathrm{H}_{2} \mathrm{O}_{2}$ and $\mathrm{NO}(+0.7$ and $+0.9 \mathrm{~V} v s . \mathrm{Ag} / \mathrm{AgCl}$ on platinum electrode, respectively) (Gifford et al., 2005), an applied electrode potential of $+0.6 \mathrm{~V} v s . \mathrm{Ag} / \mathrm{AgCl}$ was selected to monitor glucose concentration in the presence of $\mathrm{NO}$ release to avoid masking the $\mathrm{H}_{2} \mathrm{O}_{2}$ oxidation response at large $\mathrm{NO}$ fluxes (Figure $\mathrm{S} 1$ ). This potential provided the optimal combination of adequate glucose sensitivity and minimal current interference from NO oxidation. Glucose sensitivity was maintained independent of NO release from the membrane. For example, the normalized sensitivity of RSNO-MPTMS/ TEOS particle-doped $\left(0.6 \mathrm{mg} \mathrm{cm}^{-2}\right)$ polyurethane-coated glucose sensors over $3 \mathrm{~d}$ was not altered even though the NO flux from the membrane was changing appreciably. Of note, a preconditioning period of $18 \mathrm{~h}$ was required with the addition of a polyurethane barrier layer to extend the linear response (Figure S2). This change may be attributed to decreased water uptake rate by the thicker membrane, as previously observed for other polyurethane coated glucose sensors (Bindra et al., 1991; Yan et al., 2011).

The fabricated NO-releasing particle-doped membranes were characterized as having adequate selectivity over known interferents (Heller and Feldman, 2008; Wilson and Gifford, 2005). For example, glucose sensors prepared using RSNO-MPTMS/TEOS particle-doped $\left(0.6 \mathrm{mg} \mathrm{cm}^{-2}\right)$ polyurethane membranes exhibited amperometric selectivity coefficients of $0.55 \pm 0.18,0.88 \pm 0.14$, and $-0.17 \pm 0.13$ for ascorbic acid, uric acid, and nitrite over glucose, respectively.

\subsection{Variation of type of NO donors}

The NO-donor systems embedded in the 50\% w/w TPU/HPU polyurethane membranes enabled the comparison of $\mathrm{NO}$ release kinetics and payloads on sensor response. Two NO donor classes were investigated: $N$-diazeniumdiolates and $S$-nitrosothiols. Briefly, $N$ diazeniumdiolates are formed on secondary amines and produce $\mathrm{NO}$ upon decomposition by protonation in aqueous solutions (Shin et al., 2007; Wang et al., 2002). Alternatively, $S$ nitrosothiols (RSNO), endogenous transporters of NO, are formed on thiol precursors and degraded by heat, light and/or copper ions to liberate NO (Al-Sa'doni and Ferro, 2004). While NO donors may be synthesized as low molecular weight compounds or larger macromolecules (e.g., dendrimers and silica particles) (Wang et al., 2002), the following 
NO-releasing scaffolds were chosen for study because of their low toxicity, stable nature, and wide range of achievable NO-release: (1) PROLI/NO, a water soluble, $\mathrm{N}$ -

diazeniumdiolated low molecular weight compound derived from the amino acid proline; (2) three types of $N$-diazeniumdiolate-modified silica particles (i.e., MAP3/TMOS, AEAP3/ TMOS, and AHAP3/TEOS); and (3) RSNO-modified silica particles (i.e., MPTMS/TEOS). The NO release from $N$-diazeniumdiolate-modified silica particles was tuned via altering the structure of the aminosilane precursors. For example, MAP3 does not contain a stabilizing primary amine, and its decomposition rate in solution is greater than AHAP3 or AEAP3 (Shin et al., 2007; Shin and Schoenfisch, 2008). While both AEAP3 and AHAP3 contain a secondary and a primary amine, the spacing between the two amines is increased from two to six carbons resulting in altered $N$-diazeniumdiolate stability and ensuing $\mathrm{NO}$ release. The RSNO-modified silica particle scaffold enables more sustained NO release compared to the $\mathrm{N}$-diazeniumdiolate-modified silica particles as the decomposition is based on different mechanisms (thermal vs. proton) (Riccio et al., 2011).

Nitric oxide release from the particle-doped films was monitored in PBS (pH 7.4) at $37^{\circ} \mathrm{C}$ to mimic physiological conditions. Corresponding NO release data for each system studied is provided in Table 1. Due to the short half-life of PROLI/NO ( 1.2 min), 99\% of the stored $\mathrm{NO}\left(\sim 3 \mu \mathrm{mol} \mathrm{cm}{ }^{-2}\right)$ was released within the first $10 \mathrm{~min}$. The total amount of NO delivered from PROLI/NO-doped polyurethane films was greater than any particle-doped system of equivalent wt $\%$ of dopant. The AEAP3/TMOS particle-doped polyurethane composition released a similar maximum NO flux $\left(116.1 \mathrm{pmol} \mathrm{cm}^{-2} \mathrm{~s}^{-1}\right)$ and total NO amount $\left(1.70 \mu \mathrm{mol} \mathrm{cm}{ }^{-2}\right)$ as previously reported materials shown to reduce capsule formation and the chronic inflammatory response (Hetrick et al., 2007). However, the NO release duration was limited (i.e., $20 \mathrm{~h}$ ) and the NO flux was exhausted (i.e., undetectable) after $24 \mathrm{~h}$. Although MAP3/TMOS and AHAP3/TEOS particle-doped polyurethane membranes were also characterized by limited NO release durations (i.e., $\sim 15 \mathrm{~h}$ ), their maximum NO fluxes were $\sim 4$ times larger than the AEAP3/TMOS particle-doped films. The MPTMS/TEOS particle-doped films exhibited significantly longer NO release durations ( $\sim 2$ weeks) representing significantly extended release compared to previously published NO-releasing glucose sensors (Gifford et al., 2005; Shin et al., 2004). Of note, the maximum NO flux was 4 times larger (i.e., $426.2 \mathrm{pmol} \mathrm{cm}^{-2} \mathrm{~s}^{-1}$ ) than the previous NO-releasing materials shown to successfully mitigate the FBR (Hetrick et al., 2007). The NO fluxes and delivery totals indicate that each type of NO-releasing sensor membrane, regardless of NO donor type, may hold potential for improving the biocompatibility of implantable glucose sensors. The multitude of NO-releasing membranes offers a wide range of tunability to allow for diverse NO fluxes, durations, and amounts necessary for future studies aimed at exploring the in vivo response of these sensors as a function of NO release.

The analytical performance of the NO-releasing sensors fabricated with the above membranes was evaluated to assess the effects of the scaffold type and NO release on sensor response. Similar to previously reported in vivo NO-releasing glucose sensors (Gifford et al., 2005), the sensitivity and response time ( $t_{95} \%$ ) of the fabricated sensors were found to be applicable for in vivo glucose sensing (e.g., $\sim 150 \mathrm{nA} \mathrm{mM}^{-1}$ and $<1 \mathrm{~min}$, respectively). Although the permeability of PROLI/NO-doped polyurethane films to $\mathrm{H}_{2} \mathrm{O}_{2}$ and $\mathrm{O}_{2}$ was not significantly different from control membranes, glucose sensitivity was decreased by $\sim 51 \%$. We attribute this loss to the production of high local concentrations of NO affecting GOx activity (Shin et al., 2004). When larger NO-releasing scaffolds (i.e., silica particles) were used as dopants, glucose sensitivity decreased by $\sim 57 \%$ and $\sim 11 \%$ as compared to control (i.e., non-particle-doped) and PROLI/NO-doped membrane, respectively. The permeability of $\mathrm{H}_{2} \mathrm{O}_{2}$ through the particle-doped films also decreased by $\sim 58 \%$ compared to films without particles or those doped with PROLI/NO. Perhaps related, the silica particle-doped polyurethane films were significantly thicker than films without particles or with PROLI/NO 
(14 vs. $2 \mu \mathrm{m}$ thick). While larger concentrations of NO may affect glucose oxidase activity (Shin et al., 2004), the bulk concentration of NO released from these particle-doped membranes (i.e., $<100 \mu \mathrm{M}$ ) was not sufficient to alter the glucose oxidase activity as determined using an enzymatic spectroscopic assay (Bergmeyer et al., 1974). As such, the decrease in $\mathrm{H}_{2} \mathrm{O}_{2}$ diffusion and glucose sensitivity most likely results from an increase in membrane thickness. Although RSNO particles were 7 times larger in diameter than $\mathrm{N}$ diazeniumdiolated particles (Table S1), the sensitivity of the resulting sensors was not significantly altered as a function of silica particle size.

Although silica is generally regarded as nontoxic (Barbe et al., 2004), the stability of the NO-releasing dopant within the polyurethane layer was investigated to further assess the potential in vivo utility of these membranes. Silica particle leaching from the polyurethane layer into a solution mimicking physiological conditions was examined by soaking NOreleasing silica particle-doped polyurethane films in PBS (pH 7.4 at $37^{\circ} \mathrm{C}$ ). For all membrane compositions, the percent leaching was less than their respective limits of detection using DLS $(<0.6 \%$ and $<0.3 \%$ for $N$-diazeniumdiolate and RSNO-modified silica nanoparticle-doped films, respectively) after 7 days, regardless of the NO donor (Table 2).

\subsection{Variation of an additional polyurethane layer to alter NO release kinetics}

Although NO release from RSNO-functionalized particle-doped polyurethane films was unaffected by the application of an additional polyurethane layer because temperature and not water influences RSNO decomposition (data not shown), NO release from $\mathrm{N}$ diazeniumdiolated particle-doped polyurethane films was tunable via additional polymer layers to control water uptake and the resulting $N$-diazeniumdiolate decomposition. As shown in Table 3, the hydrophobic character of the polyurethane layer cast onto the NOreleasing layer restricted water access to the NO donor material, thus effectively slowing NO release. This behavior was expected based on work done previously by Frost et al. (Frost et al., 2005). When employing Tecoplast TP-470-000 PU (having the lowest water uptake) as the outer membrane, the maximum $\mathrm{NO}$ flux decreased from 77.2 to $25.7 \mathrm{pmol}$ $\mathrm{cm}^{-2} \mathrm{~s}^{-1}$ and NO release duration increased from 2.5 to $189 \mathrm{~h}$ relative to Tecophilic HP-93A-100 PU (having the highest water uptake). The time to reach the maximum NO flux was also significantly extended from $4 \mathrm{~min}$ to $5 \mathrm{~h}$. Although the extent of water uptake differed among the four types of hydrophobic/hydrophilic polyurethanes, NO-release character appeared to be bimodal (Figure S3). Both hydrophilic PUs (HP-93A-100 and AL-25-80A) exhibited fast NO release, while the more hydrophobic PUs (SG-80A and TP-470-000) had more sustained NO release. Of note, these results may be indicative of a minimum threshold of water diffusion (water uptake value between 0.2 and $0.6 \mathrm{mg} \mathrm{H}_{2} \mathrm{O} \cdot(\mathrm{mg}$ $\mathrm{PU})^{-1}$ ) necessary to decompose $N$-diazeniumdiolates. Seemingly above/below this threshold, there is little effect on NO release kinetics. Both the glucose sensitivity and $\mathrm{H}_{2} \mathrm{O}_{2}$ permeability decreased as water uptake of the additional PU layer decreased (Table 3 ). However, the dynamic range was extended from 1-6 to 1-25 mM glucose due to reduced $\mathrm{O}_{2}$ saturation. In the case where the most hydrophobic polyurethane (i.e., TP-470-000) was used to fabricate sensors, little if any glucose response was achieved due to inadequate analyte permeability (Table 3 ). Thus, we note an inverse relationship between sustained NO release and achieving sufficient glucose permeability.

To further alter NO release from the sensor membranes, the polymer concentration and thus thickness of the additional polyurethane was varied. These studies were carried out using the Tecoflex SG-80A (TPU) polymer to balance sustained NO release with adequate response to glucose. As the concentration of the TPU layer was increased from 20 to $60 \mathrm{mg} \mathrm{mL}^{-1}$ (21$46 \mu \mathrm{m}$ thick), the NO release duration and time to reach the maximum NO flux both increased. As expected, the maximum NO flux was suppressed as the diffusion of water through the membrane decreased (Fig. 1). At $60 \mathrm{mg} \mathrm{mL}^{-1} \mathrm{TPU}$, glucose response was no 
longer achievable (Table 4). The preparation of hydrophobic porous membranes via electrospun nanofibers doped with $\mathrm{NO}$ donors may prove to be a viable alternative for designing longer NO releasing functional sensors (Coneski et al., 2011).

\subsection{Variation of amount of silica particles doped within polyurethane film}

Since the RSNO-modified MPTMS/TEOS particle-doped films enable the longest NO release duration (i.e., $14 \mathrm{~d}$ ) with minimal silica leaching, this composition was chosen as a model system for studying the effect of particle concentration on NO release characteristics and sensor performance. As expected, the total amount and maximum flux of NO release were enhanced by increasing the concentration of particles doped within the polymer layer from 0.2 to $2.4 \mathrm{mg} \mathrm{cm}^{-2}$, while the casting volume was held constant (corresponding to 5 $\mu \mathrm{L}$ casting volume of $9-144 \mathrm{mg} \mathrm{mL}^{-1}$ particle solution) (Table 5). The associated NO release half-life from this film was $\sim 5 \mathrm{~h}$. Moreover, the film continued to release NO above the detection limit (i.e., $0.5 \mathrm{pmol} \mathrm{cm} \mathrm{cm}^{-2}$ ) after $14 \mathrm{~d}$ (Figure S4). The maximum NO flux and total NO release were $\sim 2560 \mathrm{pmol} \mathrm{cm}^{-2} \mathrm{~s}^{-1}$ and $9.08 \mu \mathrm{mol} \mathrm{cm}{ }^{-2}$, respectively, for membranes prepared using the largest particle concentration $\left(2.4 \mathrm{mg} \mathrm{cm}^{-2}\right)$. Although high levels of NO release may cause apoptosis, NO release from the largest particle concentration doped-membrane was lower than levels reported to be cytotoxic or apoptosis initiating $(>100 \mu \mathrm{M})($ Cals-Grierson and Ormerod, 2004; Lipton et al., 1993). Additionally, Hetrick et al. reported that NO-donor modified particles (70\% MAP3/TEOS particles) at $8 \mathrm{mg} \mathrm{mL}^{-1}$

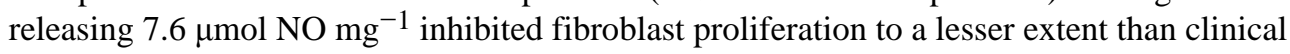
concentrations of currently administered antiseptics (e.g., chlorhexidine) (Hetrick et al., 2009). Nevertheless, the in vivo utility of these sensor membranes must next be assessed in an appropriate animal model.

As the concentration of the NO-releasing silica scaffold was increased, we observed decreased membrane stability. For example, silica particle leaching and loss of membrane integrity were observed for overloaded polymers, particularly when the particle concentration was $\sim 7$ times greater than the polymer concentration in which the particle were dissolved (i.e., $144 \mathrm{mg} \mathrm{mL}^{-1}$ particles in $20 \mathrm{mg} \mathrm{mL}^{-1} \mathrm{PU}$ solution). In addition to film instability, larger dopant concentrations hindered our ability to fabricate homogeneous films with consistent NO release. For example, larger standard deviations for maximum NO fluxes were observed for the greatest particle dopant concentrations (Table 5). At greater local concentrations of NO, the buildup of NO may facilitate accelerated RSNO decomposition from within the films due to an autocatalytic decomposition mechanism (Grossi and Montevecchi, 2002). Film thickness increased from 5.67 to $51.63 \mu \mathrm{m}$ upon increasing the particle concentration in the films from 0.2 to $2.4 \mathrm{mg} \mathrm{cm}^{-2}$, respectively, despite keeping the casting volume constant. As a result, biosensor sensitivity was decreased and response time increased with larger particle concentrations due to decreased analyte permeability (Table 5).

\section{Conclusion}

A total NO release of $\sim 1.35 \mu \mathrm{mol} \mathrm{cm} \mathrm{cm}^{-2}$ from an implant surface was previously shown to reduce the FBR (Hetrick et al., 2007). The total and maximum NO flux of the RSNOmodified MPTMS/TEOS particle-doped $\left(0.6 \mathrm{mg} \mathrm{cm}^{-2}\right)$ films presented here are 6 times greater with a 4-fold longer duration. Thus, the NO-releasing particle-doped sensor membranes presented here hold promise for improving the biocompatibility of in vivo glucose sensors and potential to improve their analytical utility. Moreover, it is clear that our design allows for tunable NO fluxes and delivery totals by altering: (1) the type of NOreleasing dopant; (2) the type of polymer utilized; (3) the concentration of additional polymer layers; and (4) the amount of dopant in the NO-releasing layer. The glucose sensor performance (i.e., sensitivity, response time, and dynamic range) was affected by these 
modifications and must be evaluated and optimized for each NO-releasing membrane composition employed in order to fall within the clinically relevant range for in vivo glucose monitoring. While Hetrick et al. (Hetrick et al., 2007) demonstrated the feasibility of a particular NO-releasing coating to mitigate the FBR, the materials reported here represent a broader range of NO donor type, polymer matrix, and NO release characteristics. A comprehensive in vivo study aimed at characterizing the FBR as a function of NO flux and duration from equivalent polyurethane membranes is currently underway. Studies evaluating any benefit of NO release on the analytical performance of implanted needle-type glucose biosensors are also underway.

\section{Supplementary Material}

Refer to Web version on PubMed Central for supplementary material.

\section{Acknowledgments}

This research was supported by the National Institutes of Health (EB000708).

\section{References}

Al-Sádoni HH, Ferro A. Curr Med Chem. 2004; 11:2679-2690. [PubMed: 15544469]

Amstad E, Gillich T, Bilecka I, Textor M, Reimhult E. Nano Lett. 2009; 9:4042-4048. [PubMed: 19835370]

Barbe C, Bartlett J, Kong LG, Finnie K, Lin HQ, Larkin M, Calleja S, Bush A, Calleja G. Adv Mater. 2004; 16:1959-1966.

Bergmeyer, HU.; Gawehn, K.; Grassl, M. Method of Enzymatic Analysis. second. Academic Press Inc; New York: 1974.

Bindra DS, Zhang Y, Wilson GS, Sterberg R, Thevenot DR, Moatti D, Reach G. Anal Chem. 1991; 63:1692-1696. [PubMed: 1789439]

Cals-Grierson MM, Ormerod AD. Nitric Oxide. 2004; 10:179-193. [PubMed: 15275864]

Chae SY, Lee M, Kim SW, Bae YH. Biomaterials. 2004; 25:843-850. [PubMed: 14609673]

Coneski PN, Nash JA, Schoenfisch MH. ACS Appl Mater Interfaces. 2011; 3:426-432. [PubMed: 21250642]

Frost MC, Reynolds MM, Meyerhoff ME. Biomaterials. 2005; 26:1685-1693. [PubMed: 15576142]

Gifford R, Batchelor MM, Lee Y, Gokulrangan G, Meyerhoff ME, Wilson GS. J Biomed Mater Res Part A. 2005; 75A:755-766.

Grossi L, Montevecchi PC. Chem Eur J. 2002; 8:380-387.

Heller A, Feldman B. Chem Rev. 2008; 108:2482-2505. [PubMed: 18465900]

Hetrick EM, Prichard HL, Klitzman B, Schoenfisch MH. Biomaterials. 2007; 28:4571-4580. [PubMed: 17681598]

Hetrick EM, Schoenfisch MH. Chem Soc Rev. 2006; 35:780-789. [PubMed: 16936926]

Hetrick EM, Schoenfisch MH. Biomaterials. 2007; 28:1948-1956. [PubMed: 17240444]

Hetrick EM, Shin JH, Paul HS, Schoenfisch MH. Biomaterials. 2009; 30:2782-2789. [PubMed: 19233464]

Hetrick EM, Shin JH, Stasko NA, Johnson CB, Wespe DA, Holmuhamedov E, Schoenfisch MH. ACS Nano. 2008; 2:235-246. [PubMed: 19206623]

Klonoff DC. Diab Care. 2005a; 28:1231-1239.

Klonoff DC. Diab Technol Ther. 2005b; 7:770-775.

Lahtinen M, Höolttä P, Riekkola ML, Yohannes G. Phys Chem Earth. 2010; 35:265-270.

Lipton SA, Choi YB, Pan ZH, Lei SZ, Chen HSV, Sucher NJ, Loscalzo J, Singel DJ, Stamler JS. Nature. 1993; 364:626-632. [PubMed: 8394509]

Nablo BJ, Chen TY, Schoenfisch MH. J Am Chem Soc. 2001; 123:9712-9713. [PubMed: 11572708] 
Nablo BJ, Prichard HL, Butler RD, Klitzman B, Schoenfisch MH. Biomaterials. 2005a; 26:69846990. [PubMed: 15978663]

Nablo BJ, Rothrock AR, Schoenfisch MH. Biomaterials. 2005b; 26:917-924. [PubMed: 15353203]

Nablo BJ, Schoenfisch MH. J Biomed Mater Res Part A. 2003; 67A:1276-1283.

Nichols SP, Le NN, Klitzman B, Schoenfisch MH. Anal Chem. 2011; 83:1180-1184. [PubMed: 21235247]

Oh BK, Robbins ME, Nablo BJ, Schoenfisch MH. Biosens Bioelectron. 2005; 21:749-757. [PubMed: $16242614]$

Privett BJ, Nutz ST, Schoenfisch MH. Biofouling. 2010; 26:973-983. [PubMed: 21082455]

Riccio DA, Dobmeier KP, Hetrick EM, Privett BJ, Paul HS, Schoenfisch MH. Biomaterials. 2009; 30:4494-4502. [PubMed: 19501904]

Riccio DA, Nugent JL, Schoenfisch MH. Chem Mater. 2011; 23:1727-1735. [PubMed: 21499510]

Saavedra JE, Southan GJ, Davies KM, Lundell A, Markou C, Hanson SR, Adrie C, Hurford WE, Zapol WM, Keefer LK. J Med Chem. 1996; 39:4361-4365. [PubMed: 8893830]

Schoenfisch MH, Rothrock AR, Shin JH, Polizzi MA, Brinkley MF, Dobmeier KP. Biosens Bioelectron. 2006; 22:306-312. [PubMed: 16483759]

Shin JH, Marxer SM, Schoenfisch MH. Anal Chem. 2004; 76:4543-4549. [PubMed: 15283600]

Shin JH, Metzger SK, Schoenfisch MH. J Am Chem Soc. 2007; 129:4612-4619. [PubMed: 17375919]

Shin JH, Privett BJ, Kita JM, Wightman RM, Schoenfisch MH. Anal Chem. 2008; 80:6850-6859. [PubMed: 18714964]

Shin JH, Schoenfisch MH. Chem Mater. 2008; 20:239-249.

Wang J. Chem Rev. 2008; 108:814-825. [PubMed: 18154363]

Wang PG, Xian M, Tang XP, Wu XJ, Wen Z, Cai TW, Janczuk AJ. Chem Rev. 2002; 102:1091-1134. [PubMed: 11942788]

Ward WK, Jansen LB, Anderson E, Reach G, Klein JC, Wilson GS. Biosens Bioelectron. 2002; 17:181-189. [PubMed: 11839471]

Williams DLH. Org Biomol Chem. 2003; 1:441-449. [PubMed: 12926240]

Wilson GS, Gifford R. Biosens Bioelectron. 2005; 20:2388-2403. [PubMed: 15854814]

Wilson GS, Hu YB. Chem Rev. 2000; 100:2693-2704. [PubMed: 11749301]

Wisniewski N, Moussy F, Reichert WM. Fresenius J Anal Chem. 2000; 366:611-621. [PubMed: 11225773]

Wisniewski N, Reichert M. Colloids Surf. 2000; B18:197-219.

Yan Q, Major TC, Bartlett RH, Meyerhoff ME. Biosens Bioelectron. 2011; 26:4276-4282. [PubMed: 21592764] 

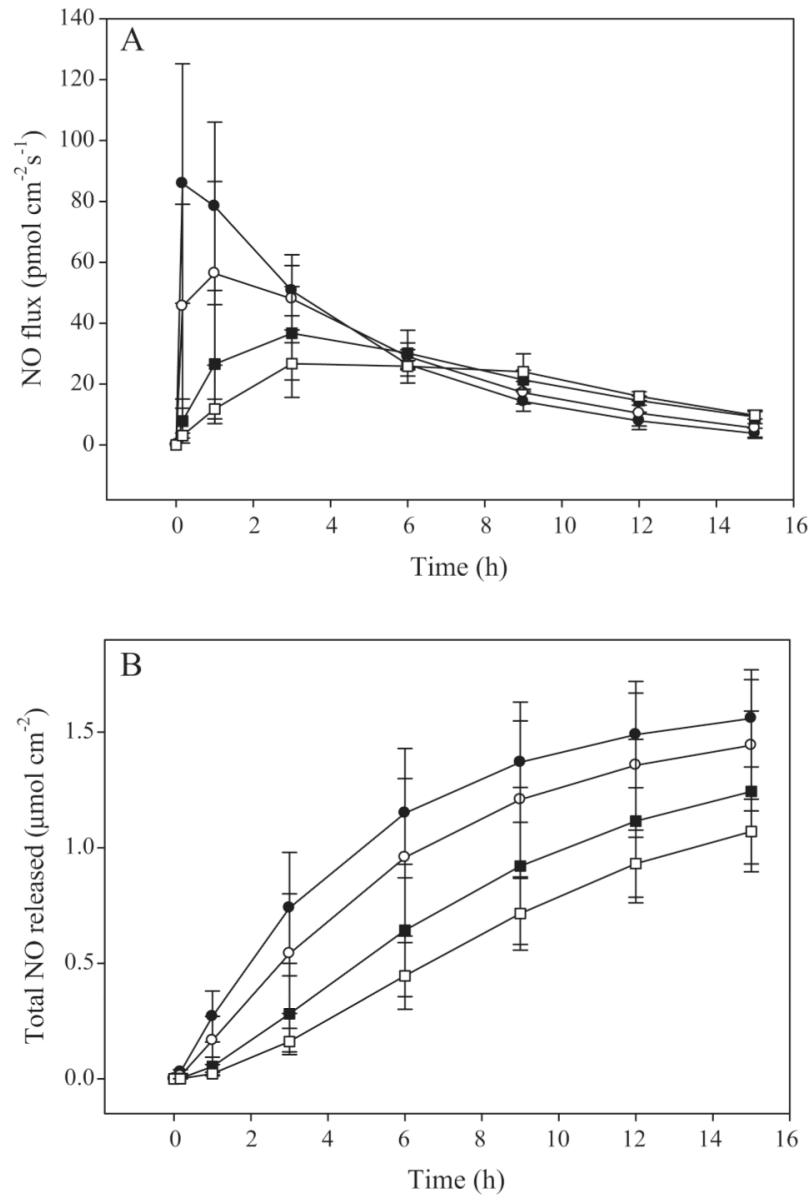

Fig. 1.

(A) Nitric oxide flux and (B) NO totals from AEAP3/TMOS particle-doped $\left(0.6 \mathrm{mg} \mathrm{cm}^{-2}\right)$ PU films (•) without and with additional Tecoflex SG-80A (TPU) layer at (०) 20, (•) 40, and () $60 \mathrm{mg} \mathrm{mL}^{-1}$ concentration. 


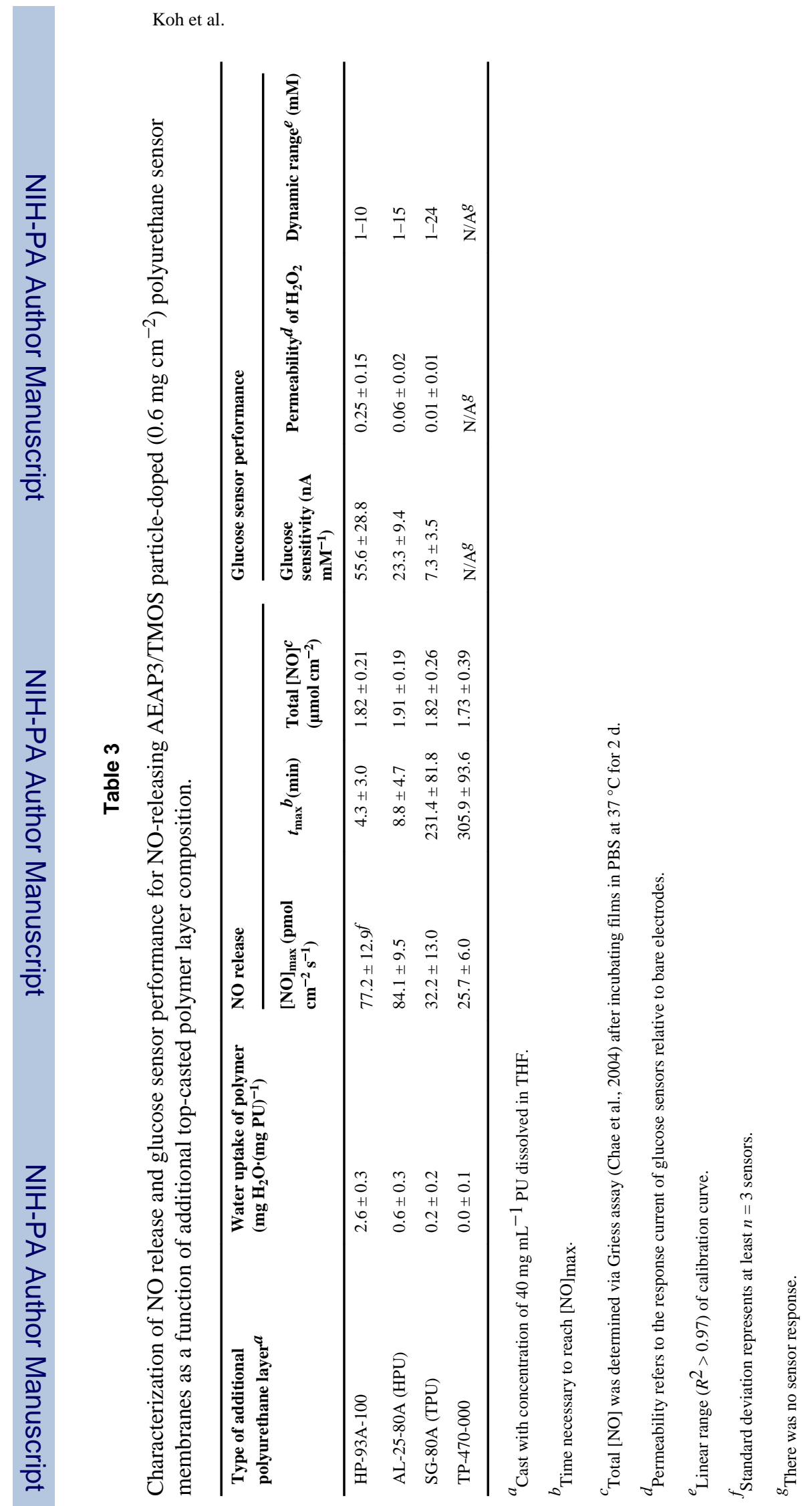

Biosens Bioelectron. Author manuscript; available in PMC 2012 October 15. 


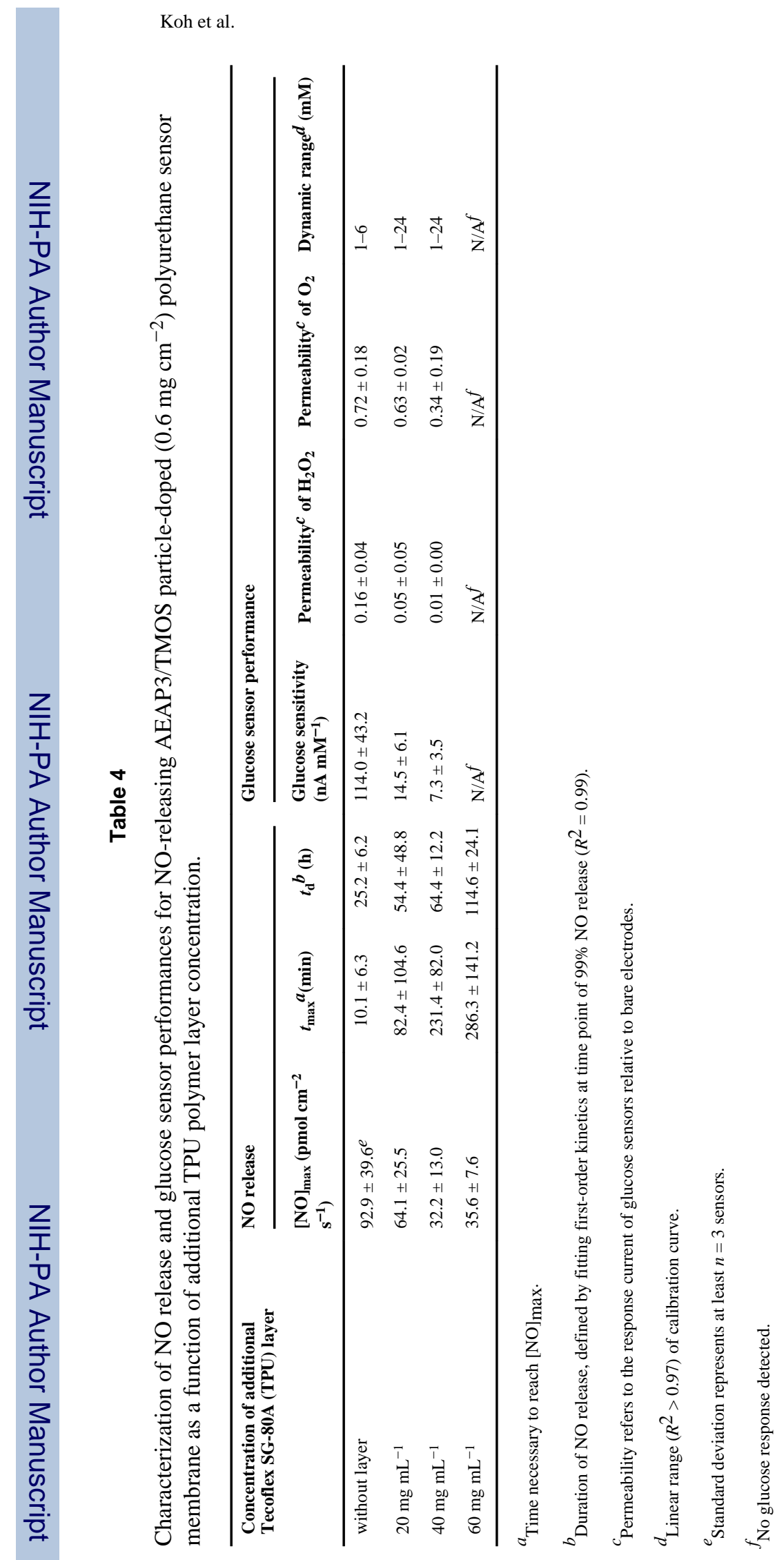

Biosens Bioelectron. Author manuscript; available in PMC 2012 October 15. 


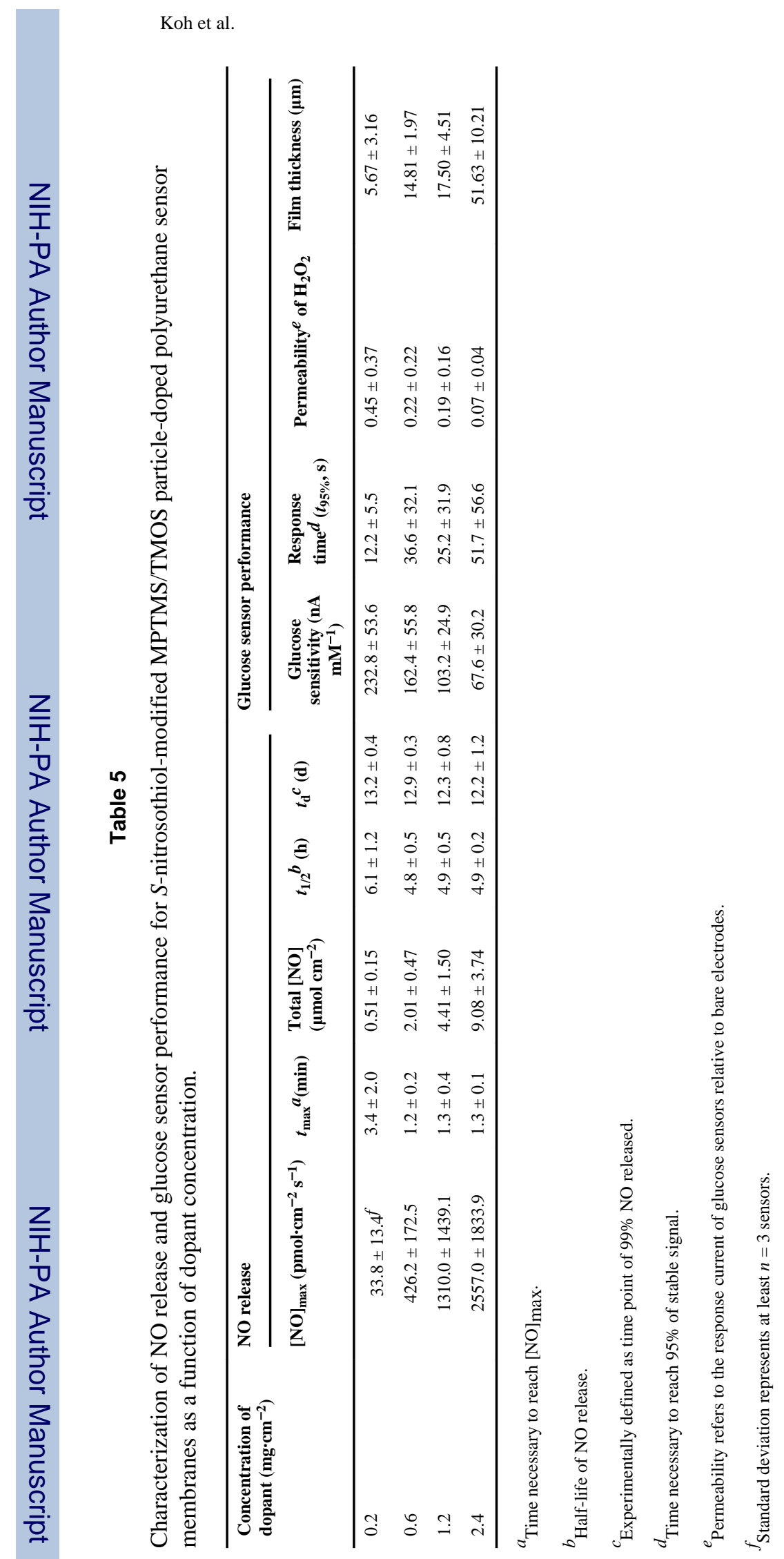

Biosens Bioelectron. Author manuscript; available in PMC 2012 October 15. 